Scientific Paper

\title{
Detailed Monte Carlo analysis of the secondary photons coming out of the therapeutic X-ray beam of linear accelerator
}

\author{
Mohamed BENCHEIKHa, ${ }^{a,}$, Abdelmajid MAGHNOUJ ${ }^{b}$, Jaouad TAJMOUATI ${ }^{b}$, Abdessamad DIDI ${ }^{c}$, Abdesslam \\ LAMRABET ${ }^{\mathrm{b}}$ \\ ${ }^{a}$ Physics Department, Faculty of Sciences and Technologies Mohammedia, Hassan II University of Casablanca, Mohammedia, \\ Morocco \\ ${ }^{b}$ LISTA Laboratory, Physics Department, Faculty of Sciences Dhar El-Mahraz, University of Sidi Mohamed Ben Abdellah, \\ Fez, Morocco \\ ${ }^{c}$ Theoretical and Particle Physics and Modelling Laboratory, Faculty of Sciences, Mohammed 1st University, Oujda, Morocco \\ *E-mail address: bc.mohamed@gmail.com
}

\begin{abstract}
External photon beam radiotherapy is often used in tumor treatment. The photons are generated from the target which had stricken by the primary electron beam (incident particles). The photon beam contains the primary photons coming directly from the target and secondary photons coming from the photon interactions with head component materials (scattered photons). Altogether is thereafter used in radiotherapy treatment. This Monte Carlo study aims to investigate and evaluate the secondary radiations (photons) in terms of fluence, energy fluence, spectral distribution, mean energy and angular spread distribution.

The secondary photons, which contributed in radiotherapy treatment, are examined and evaluated in number (fluence) and energy. At the phantom surface, the secondary photons originated in the whole linac head are mainly coming from the primary collimator. In $0.45 \%$ of secondary photons coming from the whole linac head, the primary collimator contributes by $86 \%$ and they are more energetic. However, the flattening filter and the secondary collimator contribute together by less than $14 \%$ and their photons are less energetic and then can deteriorate the beam dosimetry quality. To improve the radiotherapy treatment quality, the number of photons of low energy should be as low as possible in the clinical beam. Our work can be a basic investigation to use in the improvement of linac head configuration and specially the beam modifiers.
\end{abstract}

Key words: beam modifiers; Monte Carlo simulation; secondary photons; BEAMnrc code; BEAMDP code.

\section{Introduction}

For high quality of external photon beam radiotherapy, treatment must be used by a high number of photons, which are homogenates in energy, without losing the radiotherapy efficiency and organs at risk protection. ${ }^{1}$ Photon beam quality is ensured by instantaneous control and survey using detectors. ${ }^{2}$ At patient skin, photon beam is formed by the primary photons and the secondary photons (or scattered photons) and other particles contamination as electrons, positrons and neutrons. In this study, we are focused on the secondary photons that were produced by photon interactions with beam modifier materials, and they are investigated in terms of the fluence, energy fluence, spectral distribution and angular distribution. This work is carried out by the Monte Carlo method as a basic technique using BEAMnrc code and BEAMDP code. ${ }^{3}$ The knowledge of secondary photons' characteristics is crucial for improving and enhancing the beam modifier in the material of construction and geometry for linac development by minimizing the number and energy of scattered photons at the phantom surface.

Monte Carlo simulation is a technique that provides both accurate and detailed energetic investigation as spectral distribution and fluence evaluations and dosimetric calculation as percentage depth dose output of linear accelerator. ${ }^{4,5}$ It has been used extensively in medical physics for modeling linear accelerators and for radiation therapy dose calculation. ${ }^{6,7}$ Previously, the sensitivity of the megavoltage photon beam according to electron energy above the target and other parameters was studied. ${ }^{8}$

For high external photon beam radiotherapy efficiency, many works have studied the particles contaminations., ${ }^{9,10}$ For increasing radiotherapy quality, the secondary photons should therefore be reduced under a flattening filter, which is essential in linac head, and it should not be removed in large irradiation field size for not deteriorating the beam quality. ${ }^{11,12}$ However, 
the removal of this component affects slightly dosimetry quality for small field size..$^{5,13}$

This work is a quantitative and qualitative study of fluence and energy distributions of secondary photons originated in the beam modifiers. The objective of this work is to investigate the secondary photons (scattered photons) originated in the primary collimator (PC), flattening filter (FF), and secondary collimator (SC) of a linear accelerator for an eventual beam modifier improvement by using the Monte Carlo method. ${ }^{14,15}$

\section{Materials and Methods}

Monte Carlo geometry of Varian Clinac 2100 is built for a $6 \mathrm{MV}$ photon beam using BEAMnrc code ${ }^{16}$ and the physics process of simulation is based on EGSnrc code for simulating the particles transport as realistically as possible. ${ }^{17}$

The Monte Carlo simulation of the linac was performed based on manufacturer-provided data (Varian Medical System) using histories number of $2 \times 10^{7}$ with a Directional Bremsstrahlung Splitting (DBS) option of 1000. This number was sufficient to generate a simulation statistical uncertainty under $1 \%$.

The secondary photons are studied and investigated for a field size of $10 \times 10 \mathrm{~cm}^{2}$, the events are collected inside the square shape (square surface defined by the filed size) perpendicular to the beam axis. The source-to-surface distance (SSD) of $100 \mathrm{~cm}$ where the phase space file (PSF) is generated by BEAMnrc code and it contained the particles' information then it used by BEAMDP code to extract the photons' characteristics. ${ }^{18}$

Figure 1 gives the cross-view of linac head geometry to simulate by BEAMnrc and the scoring plan position to generate the PSF. Figure 1 shows a cross-section view of linac head geometry including PC, FF, and SC. The Monte Carlo simulation of linac was validated by accuracy according to IAEA protocols. ${ }^{19,20}$ The gamma index acceptance rate is $99 \%$ for percentage depth dose (PDD) and is $98 \%$ for beam dose profiles. $^{21}$

The secondary photons emergent from the beam modifiers; primary collimator (PC), flattening filter (FF), and secondary

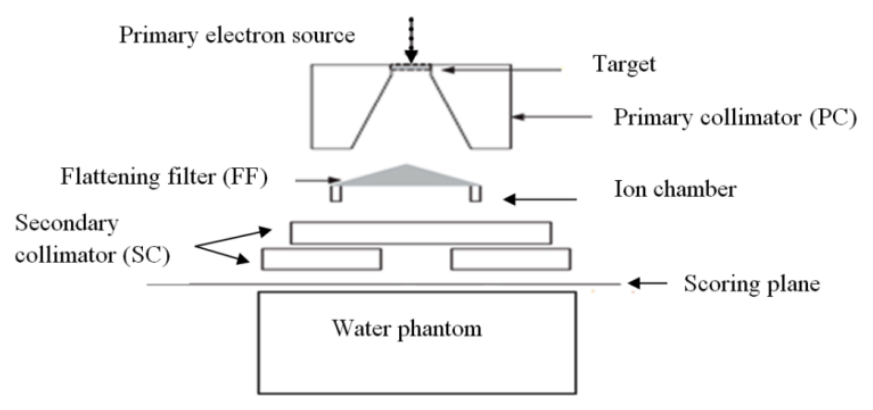

Figure 1. Cross-section view of Monte Carlo geometry of linac head and PC, FF, SC and scoring plane for phase space file collimator (SC); are investigated and their fractional contribution (FC) for each one regarding total secondary photons in the whole linac head is evaluated. The FC is determined according to the following formula:

FC $(\%)=100 \times \frac{\text { Characteristic of beam modifier }}{\text { Characteristic of the whole linac head }}$

$\mathrm{Eq} \cdot 1$

Where;

Characteristic: fluence profile, energy fluence profile, spectral distribution and angular distribution of secondary photons at the phantom surface.

The dose measurements, used in Monte Carlo simulation validation, are performed as recommended by the Swiss Society of Radiobiology and Medical Physics experiment protocols. ${ }^{22}$ However, the measurement parameters as uncertainties, temperature, pressure ... are set according to IAEA recommendations. The PDDs and dose profiles are measured using ionization chamber PTW. The overall measurements uncertainty is less than $2 \%$ as recommended by IAEA protocols. ${ }^{19,20}$

\section{Results and discussion}

\section{Fluence profile}

Fluence profile describes the photon number as a function of offaxis distance. Figure 2 shows the planar fluence profile variation of secondary photons (scattered photons) and total photons (scatter + direct) as a function of off-axis distance.

We notice from Figure 2, the fluence of secondary photons (scattered photons) is low inside the irradiation field, but in the out-of-field region, the fluence of secondary photons is higher in comparison to total photons fluence. This among of secondary photons is an integral part of the clinical beam used in the radiotherapy treatment and it can have a negative impact on treatment quality and especially for deep tumor sterilization. The question can be put in this context: where these secondary photons (scattered photons) are from and what is their FC in total secondary photons originated in the whole linac head?

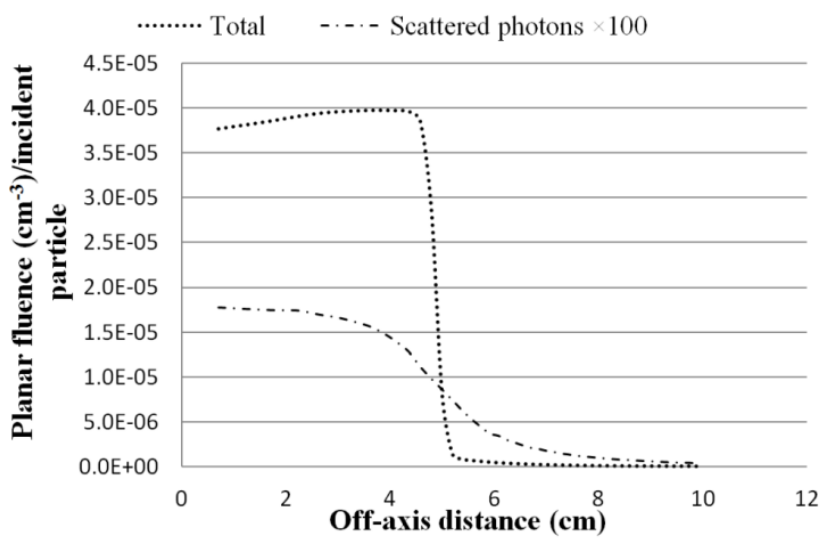

Figure 2. Fluence profile of secondary photons originated in the whole linac head and fluence of total photons as a function of offaxis distance 


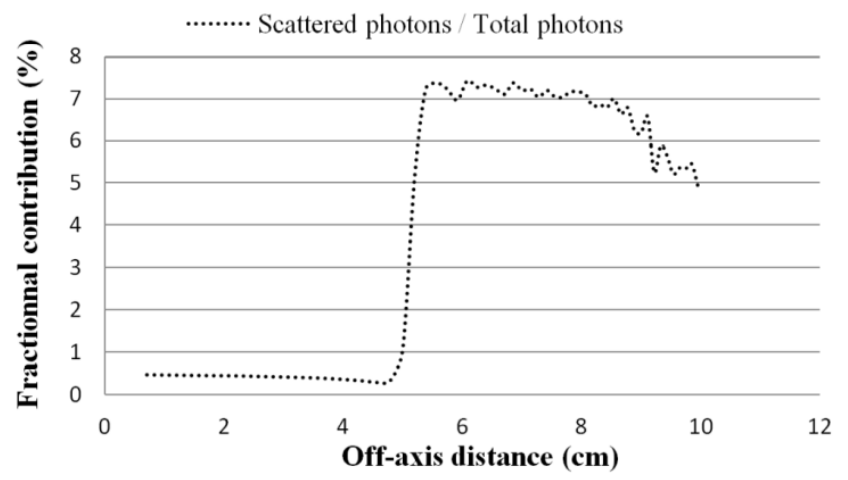

Figure 3. Fractional contribution (FC) of fluence of secondary photons in fluence of total photons as a function of off-axis distance

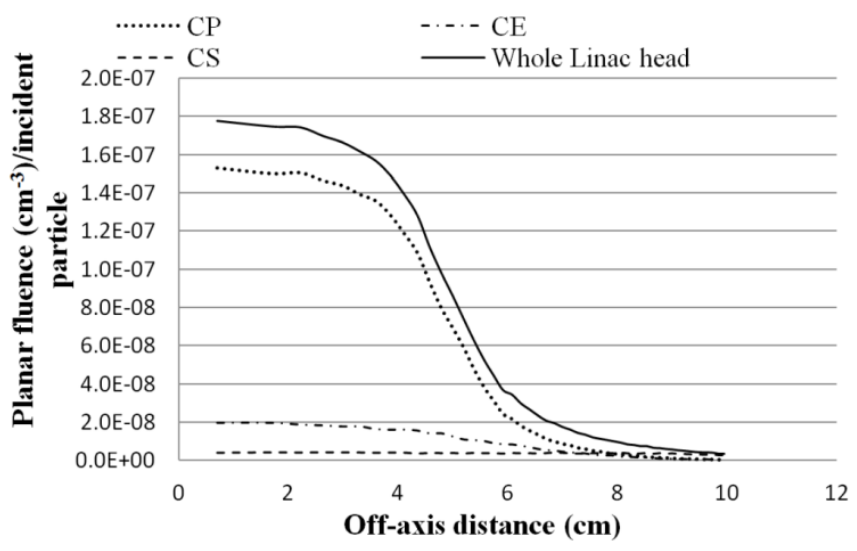

Figure 5. Fractional contribution (FC) of fluence of secondary photons originated in each beam modifier in fluence of secondary photons of the whole linac head as a function of off-axis distance

Figure 3 shows the FC of secondary photons (scattered photons) fluence in total photons fluence as a function of off-axis distance.

FC of secondary photons decreases slightly with off-axis distance and attains the minimum of $0.28 \%$ at $4.58 \mathrm{~cm}$ and increases rapidly with off-axis and attains a maximum of $7.23 \%$ at $5.38 \mathrm{~cm}$ (Figure 3). Therefore, this quantity of secondary photons will be studied and examined in terms of the fluence, energy fluence, spectral distribution, and angular distribution for beam modifiers: primary collimator (PC), flattening filter (FF) and secondary collimator (SC).

Figure 4 shows planar fluence profiles of secondary photons originated in each beam modifier beside planar fluence profiles of photons originated in the whole linac head as a function of off-axis distance.

We notice from Figure 4 that the fluence of secondary photons originated in PC is higher near the central beam axis compared to the fluence of those originated in FF and SC. Another remark is that the fluence of secondary photons decreases with off-axis distance for PC and FF but it remains constant for SC (Figure 4).

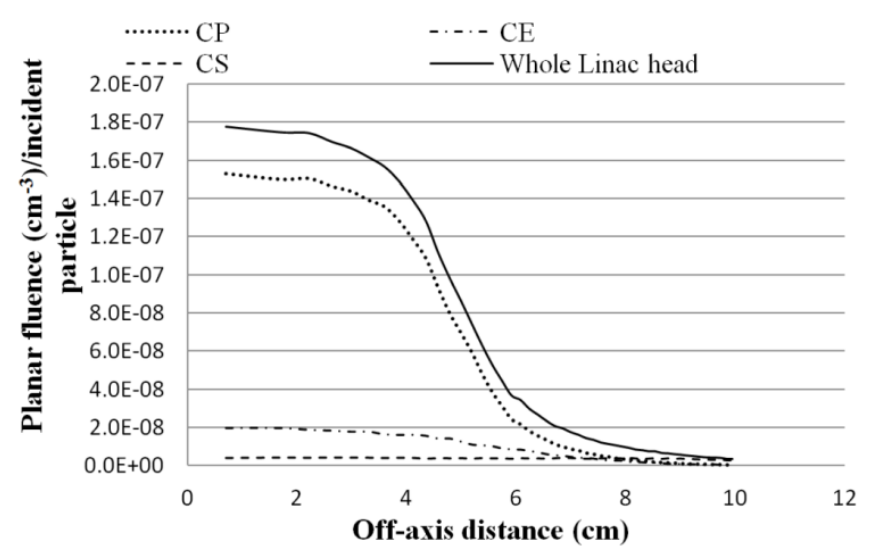

Figure 4. Planar fluence profiles of secondary photons originated in each beam modifier and secondary photons of the whole linac head as a function of off-axis distance

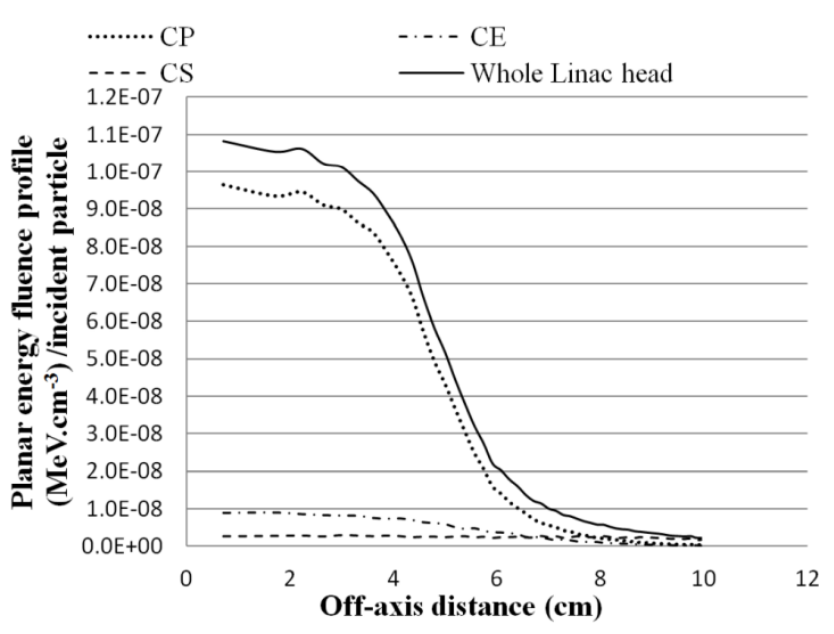

Figure 6. Energy fluence profiles of secondary photons originated in each beam modifier and secondary photons of the whole linac head as a function of off-axis distance

For good interpretation of secondary photons fluence profile results, Figure 5 shows FC of secondary photons fluence of each beam modifier in the fluence of photons of the whole linac head.

It can be seen from Figure 5, the FC for PC is higher near the central beam axis and is more than $86 \%$, but for FF and SC together, it is less than $13.5 \%$. The secondary photons originated in the whole linac head are mainly coming from PC. In theee out-of-field region where absolute fluence is quite small, the fluence of secondary photons in the whole linac head is formed by photons originated in SC beside the penumbra photons and leakage photons. ${ }^{23}$

\section{Energy fluence profile}

Energy fluence profile describes the variation of photons energy as a function of off-axis distance. Figure 6 shows energy fluence profiles of secondary photons originated in each beam modifier and secondary photons of the whole linac head as a function of off-axis distance. 
Energy fluence profiles variation is as fluence profiles shown above. Figure 7 shows FC of energy fluence of secondary photons originated in each beam modifier in fluence of secondary photons of the whole linac head.

We notice from Figure 7 that the FC of energy fluence of secondary photons originated in $\mathrm{PC}$ is higher near the central beam axis and it is approximately $90 \%$ for PC and is less than $11.5 \%$ for FF and SC together. It means that the energetic photons that are scattered in the whole linac head are mainly coming from PC (Figures 5 and 7).

\section{Spectral distribution}

Spectral distribution describes the variation of the number of photons as a function of energy; the knowledge of the spectral distribution of secondary photons is crucial for beam modifier development because photons of low energy contaminate the photon beam dosimetry. Figure 8 shows the spectral distributions of secondary photons originated in each beam modifier and secondary photons in the whole linac head.

All spectral distributions curves have a maximum around $0.49 \mathrm{MeV}$, which is $3.16 \times 10^{-7}$ photon/MeV/incident particle for $\mathrm{PC}, 2.29 \times 10^{-8}$ photon/MeV/incident particle for $\mathrm{FF}$ and $8.64 \times 10^{-9}$ photon/MeV/incident particle for $\mathrm{SC}$; but it is $1.43 \times 10^{-7}$ photon/MeV/incident particle for the whole linac head (Figure 8).

Figure 9 shows FC of spectral distribution for each beam modifier in spectral distribution for the whole linac head as a function of energy.

The FC of the spectral distribution of secondary photons originated in $\mathrm{PC}$ is higher and has a maximum of $221 \%$ at 0.49 $\mathrm{MeV}$; but it is low for FF and SC and it has a minimum of $16.1 \%$ for $\mathrm{FF}$ at $0.49 \mathrm{MeV}$ and of $4.74 \%$ for $\mathrm{SC}$ at $0.21 \mathrm{MeV}$ (Figure 9).

\section{Angular spread distribution}

Angular spread distribution describes the variation of the number of photons as a function of angular spread. Figure 10 shows the angular spread distribution of secondary photons originated in each beam modifier and secondary photons in the whole linac head.

We notice from Figure 10, the maximum of angular spread distribution decreases according to the angular degree. It is $2.05 \times 10^{-6}$ photon/degree/incident particle at $2.6^{\circ}$ for $\mathrm{PC}$, $2.59 \times 10^{-7}$ photon/degree/incident particle at $3.4^{\circ}$ for $\mathrm{FF}, 9.1 \times 10^{-}$ ${ }^{8}$ photon/degree/incident particle at $8.2^{\circ}$ for SC and $2.33 \times 10^{-6}$ photon/degree/incident particle at $2.6^{\circ}$ for the whole linac head (Figure 10).

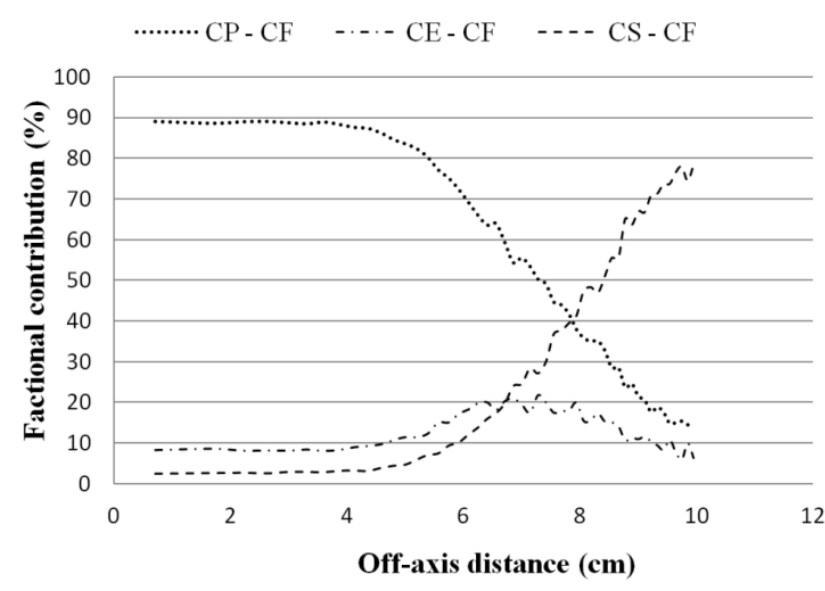

Figure 7. Fractional contribution (FC) of energy fluence of secondary photons originated in each beam modifier in energy fluence of secondary photons of the whole linac head as a function of off-axis distance

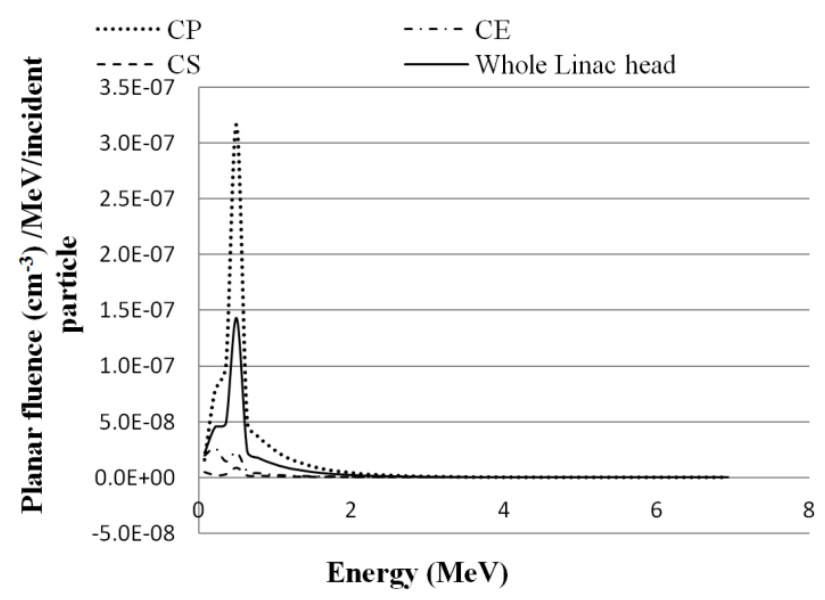

Figure 8. Spectral distributions of secondary photons originated in each beam modifier and secondary photons in the whole linac head as a function of energy

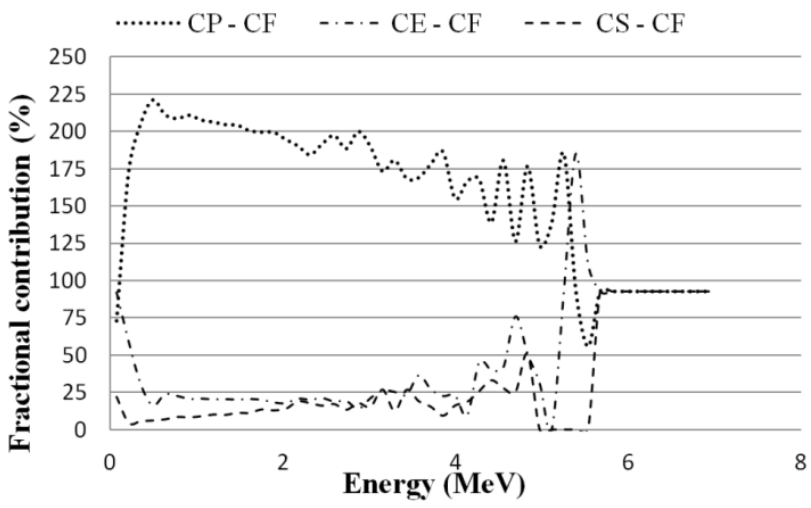

Figure 9. Fractional contribution (FC) of the spectral distribution of secondary photons originated in each beam modifier in the spectral distribution of the whole linac head as a function of energy 


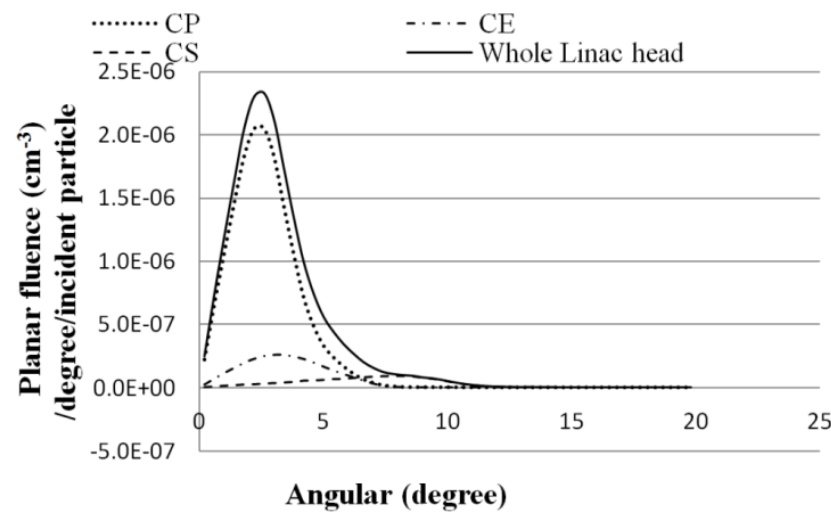

Figure 10. Angular spread distribution of secondary photons originated in each beam modifier and secondary photons in the whole linac head as a function of an angular degree

Figure 11 shows FC of angular spread distribution for each beam modifier in angular spread distribution for the whole linac head.

We notice from Figure 11, for PC, the FC decreases with an angular degree and attains a minimum of $8.6 \%$ and then increases slightly. For SC, it increases with angular degree and attains a maximum of $94 \%$ and then decreases. For FF, the FC increases with angular degree and attains a maximum of $30.8 \%$ and then decreases (Figure 11). Angular spread distribution varies inside two angular degree intervals, the first one is from $0^{\circ}$ to $6.6^{\circ}$, it increases for FF and SC but decreases for PC, the second one is from $6.6^{\circ}$, it increases for PC but decreases for FF and $\mathrm{SC}$ with angular degree.

\section{Conclusion}

Beam modifiers are a potential source of secondary radiation (photons) in the linac head. At the phantom surface, the secondary photons originated in the whole linac head are mainly coming from PC; the FF removes the photons of low energy and lets the energetic ones reach the patient and contribute to the

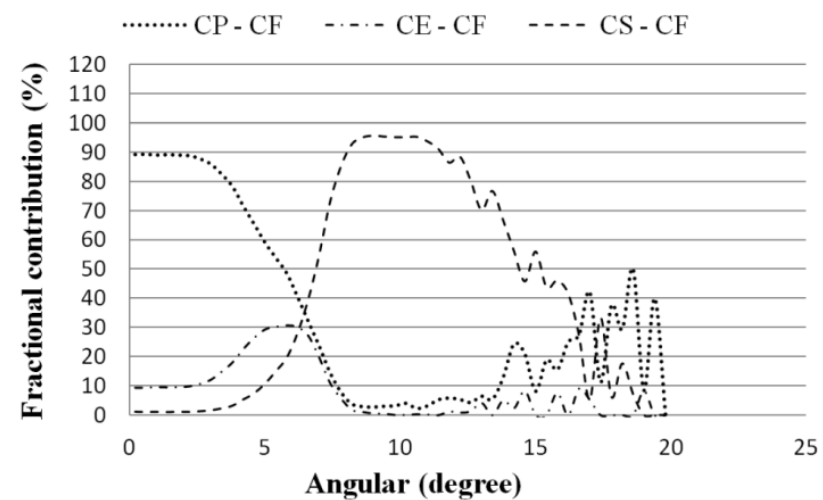

Figure 11. Fractional contribution (FC) of angular spread distribution of secondary photons originated in each beam modifier in angular spread distribution in the whole linac head as a function of an angular degree

radiotherapy treatment. Under FF, this component and SC produced a low number of the secondary photons of low energy, which makes the beam contamination lower, and the quality may even deteriorate if the material used in FF construction is more sensitive to Compton effect.

If $\mathrm{FF}$ is removed from the linac head, the photon beam will be more contaminated by the photons of low energy and the dosimetry quality is thereafter deteriorated. ${ }^{24}$ The findings of our study are more significant than the previous one done by Chaney et al. ${ }^{25,26}$ We have previously studied the flattening filter design by analyzing the photon beam softening as a physical characterization for an eventual dosimetry quality improvement. ${ }^{27,28}$

\section{Acknowledgement}

As authors, we would like to thank Varian Medical System for providing us the Varian Clinac 2100 geometry data and giving us this opportunity to study the Varian linear accelerator technology and take part in its future development.

\section{References}

1. Hill R, Healey B, Holloway L, et al. Advances in kilovoltage x-ray beam dosimetry. Phys Med Biol. 2014;59(6):R183-231. https://doi.org/10.1088/0031-9155/59/6/R183

2. Seuntjens JP, Ross CK, Shortt KR, Rogers DW O. Absorbed-dose beam quality conversion factors for cylindrical chambers in high energy photon beams. Med Phys. 2000:27(12): 2763-2779. https://doi.org/10.1118/1.1328081

3. Chetty IJ, Curran B, Cygler JE, et al. Report of the AAPM Task Group no 105: Issues associated with clinical implementation of Monte Carlo based photon and electron external beam treatment planning. Med Phys. 2007;34(12):4818-4853. https://doi.org/10.1118/1.2795842

4. Verhaegen F, Seuntjens J. Monte Carlo modelling of external radiotherapy photon beams. Phys Med Biol. 2003;48(21):R107-164. https://doi.org/10.1088/0031-9155/48/21/r01

5. Bencheikh M, Maghnouj A, Tajmouati J. Energetic Properties' Investigation of Removing Flattening Filter at Phantom Surface: Monte Carlo Study using BEAMnrc Code, DOSXYZnrc Code and BEAMDP Code. PEPAN Letters. 2017;14(6):921-930. https://doi.org/10.1134/S1547477117060073

6. Bencheikh M, Maghnouj A, Tajmouati J. Photon beam softening coefficient determination with slab thickness in small filed size: Monte Carlo study. PEPAN Letters, 2017:14(6):685-686 
7. Didi A, Dadouch A., Bencheikh M, Jai O. Monte Carlo simulation of thermal neutron flux of americium-beryllium source used in neutron activation analysis. Moscow University Physics Bulletin. 2017;72(5):460-464. https://doi.org/10.3103/S0027134917050022

8. Sheikh-Bagheri D, Rogers DWO. Sensitivity of megavoltage photon beam Monte Carlo simulations to electron beam and other parameters. Med Phys. 2002;29(3):379-390. https://doi.org/10.1118/1.1446109

9. Vega-Carrillo HR, Martınez-Ovalle SA, Lallena AM, et al. Neutron and photon spectra in LINACs. Applied Radiation and Isotopes. 2012;71(Supplement):75-80. https://doi.org/10.1016/j.apradiso.2012.03.034

10. Medina AL, Teijeiro A, Salvador F, et al. Comparison between TG-51 and TRS-398: electron contamination effect on photon beamquality specification. Phys Med Biol. 2004;49(1):17-32. https://doi.org/10.1088/0031-9155/49/1/002

11. Bencheikh M, Maghnouj A, Tajmouati J. Photon beam softening coefficients evaluation for a $6 \mathrm{MeV}$ photon beam for an aluminum slab: Monte Carlo study using BEAMnrc code, DOSXYZnrc code and BEAMDP code. Moscow University Physics Bulletin. 2017;72(3):263-270. https://doi.org/10.3103/S0027134917030043

12. Bencheikh M, Maghnouj A, Tajmouati J. Dosimetry Investigation and Evaluation for Removing Flattening Filter Configuration of linac: Monte Carlo Study. Moscow University Physics Bulletin. 2017;72(6):640-646. https://doi.org/10.3103/S0027134918660025

13. Bencheikh M, Maghnouj A, Tajmouati J. Study of photon beam dosimetry quality for removing flattening filter linac configuration. Annals of University of Craiova Physics AUC. 2017;27:50-60.

14. Reynaert N, van der Marck SC, Schaart DR, et al. Monte Carlo treatment planning for photon and electron beams. Rad Phys Chem. 2017;76(4):643-686. https://doi.org/10.1016/j.radphyschem.2006.05.015

15. Pearson D, Parsai E, Fledmeier J. SU-FF-T-223: Evaluation of dosimetric properties of 6 and $10 \mathrm{MeV}$ photon beams from a linear accelerator with no flattening filter. Med Phys;33:2099. https://doi.org/10.1118/1.2241143

16. Rogers DWO, Walters B, Kawrakow I. BEAMnrc Users Manual. NRCC Report; Ottawa. 2013;12-254.

17. Rogers DWO, Kawrakow I, Seuntjens JP, et al. NRC User Codes for EGSnrc. NRCC Report; Ottawa. 2013;6-83.

18. Ma CM, Rogers DWO. BEAMDP Users Manual. NRCC Report; Ottawa. 2013;3-24.

19. IAEA Technical Reports Series No.430. Commissioning and Quality Assurance of Computerized Planning Systems for Radiation Treatment of Cancer. International Atomic Energy Agency; Vienna. 2004.

20. IAEA-TECDOC-1540. Specification and Acceptance Testing of Radiotherapy Treatment Planning Systems. International Atomic Energy Agency; Vienna. 2007.

21. Bencheikh M, Maghnouj A, Tajmouati J. Validation of Monte Carlo simulation of 6 MV photon beam produced by Varian Clinac 2100 linear accelerator using BEAMnrc code and DOSXYZnrc code. PEPAN Letters. 2017;14(5):780-787. https:// doi.org/10.1134/S154747711705003X

22. Swiss Society of Radiobiology and Medical Physics SSRMP. Dosimétrie des faisceaux de photons de haute énergie l'aide de chambres d'ionisation. SSRMP Recommandation $N^{\circ}$ 8. 2000. ISBN 3-908125-26-X.

23. García-Garduño OA, Celis MA, Lárraga-Gutiérrez JM, et al. Radiation transmission, leakage and beam penumbra measurements of a micro-multileaf collimator using GafChromic EBT film. Journal of Applied Clinical Medical Physics. 2008;9(3):90-98. https://doi.org/10.1120/jacmp.v9i3.2802

24. International Atomic Energy Agency. Absorbed dose determination in external beam radiotherapy. Technical reports series no. 398. International Atomic Energy Agency; Vienna. 2000;110-133.

25. Chaney EL, Cullip TJ, Gabriel TA. A Monte Carlo study of accelerator head scatter. Med Phys. 1994;21(9):1383-1390. https://doi.org/10.1118/1.597194

26. Zhu TC, Bjärngard BE. Head scatter off-axis for megavoltage $x$ rays. Med Phys. 2003;30(4):533-543. https://doi.org/10.1118/1.1556609

27. Bencheikh M, Maghnouj A, Tajmouati J. Study of Possibility to Reduce Flattening Filter Volume for Increasing Energetic Photons for High Radiotherapy Efficiency. Moscow University Physics Bulletin. 2017;72(6):653-657. https://doi.org/10.3103/S0027134918660049

28. Bencheikh M, Maghnouj A, Tajmouati J. Relative Attenuation and Beam Softening Study with Flattening Filter Volume Reduction: Monte Carlo Study. Moscow University Physics Bulletin. 2017;72(6):647-652. https://doi.org/10.3103/S0027134918660037 